\title{
Normal phase and superconducting instability in attractive Hubbard model: the DMFT(NRG) study
}

\author{
N.A. Kuleeva ${ }^{1 *}$, E.Z. Kuchinskii ${ }^{1} \dagger$, M.V. Sadovskii ${ }^{1,2} \ddagger$ \\ ${ }^{1}$ Institute for Electrophysics, Russian Academy of Sciences, Ural Branch, Ekaterinburg 620016, Russia \\ ${ }^{2}$ Institute for Metal Physics, Russian Academy of Sciences, Ural Branch, Ekaterinburg 620990, Russia
}

\begin{abstract}
We study the normal (non-superconducting) phase of attractive Hubbard model within dynamical mean field theory (DMFT) using numerical renormalization group (NRG) as impurity solver. Wide range of attractive potentials $U$ is considered, from the weak-coupling limit, where superconducting instability is well described by BCS approximation, up to the strong-coupling region, where superconducting transition is described by Bose-condensation of compact Cooper pairs, which are formed at temperatures much exceeding superconducting transition temperature. We calculate density of states, spectral density and optical conductivity in the normal phase for this wide range of $U$, including the disorder effects. Also we present the results on superconducting instability of the normal state dependence on the attraction strength $U$ and the degree of disorder. Disorder influence on the critical temperature $T_{c}$ is rather weak, suggesting in fact the validity of Anderson theorem, with the account of the general widening of the conduction band due to disorder.
\end{abstract}

\section{INTRODUCTION}

The studies of superconductivity in the strong coupling region attracts theorists for rather long time [1] and most important advance here was made by Nozieres and Schmitt-Rink 22], who proposed an effective approach to describe crossover from weak coupling BCS limit to the picture of Bose-Einstein condensation (BEC) of preformed Cooper pairs in the strong coupling limit. The recent progress of experimental studies of ultracold gases in magnetic and optical traps, as well as in optical lattices, allowed the controlled change of parameters, such as density and interaction strength (see reviews [3, 4] ), increasing theoretical interests for studies of superfluidity (superconductivity) for the case of very strong pairing interaction, as well as in BCS-BEC crossover region. Probably the simplest model allowing theoretical studies of BCS-BEC crossover is the attractive Hubbard model. It is widely used also for the studies of superconductor insulator transition (see review in [5]). The most effective modern approach to the solution of Hubbard model, both for strongly correlated electronic systems (SCES) with repulsive interaction and for the studies of BCSBEC crossover in the case of attraction is the dynamical mean field theory (DMFT), giving an exact solution in the limit of infinite dimensions [ 6 - 8$]$. The attractive Hubbard model was studies within DMFT in a number of recent papers $[9-12]$. However only few results were obtained for the normal (non-superconducting) phase of this model, e.g. there were practically no studies of twoparticle properties, such as optical conductivity.

To describe electronic properties of SCES we obviously need to take into account different additional interactions (electron-phonon interaction, scattering by fluctu-

\footnotetext{
*E-mail: strigina@iep.uran.ru

†E-mail: kuchinsk@iep.uran.ru

${ }^{\ddagger}$ E-mail: sadovski@iep.uran.ru
}

ations of different order-parameters, disorder scattering etc), which are inevitably present in such systems. Recently we have proposed the generalized DMFT $+\Sigma$ approach [13 16], which is very convenient and effective for the studies of such additional (external with respect to Hubbard model itself) interactions (e.g. pseudogap fluctuations [13-16], disorder [17, 18] and electron-phonon interaction [19]). This approach was also successfully extended to the analysis of optical conductivity [17, 20]. In this paper we apply the DMFT $+\Sigma$ approach to the studies of the normal state properties of attractive Hubbard model, including the effects of disorder.

\section{THE BASICS OF DMFT $+\Sigma$ APPROACH}

In general case we shall consider non-magnetic Hubbard model with site disorder. The Hamiltonian of this model can be written as:

$$
H=-t \sum_{\langle i j\rangle \sigma} a_{i \sigma}^{\dagger} a_{j \sigma}+\sum_{i \sigma} \epsilon_{i} n_{i \sigma}+U \sum_{i} n_{i \uparrow} n_{i \downarrow},
$$

where $t>0$ is the transfer integral between nearest sites of the lattice, $U$ is the onsite interaction (for the case of attraction $U<0), n_{i \sigma}=a_{i \sigma}^{\dagger} a_{i \sigma}$ is onsite electron number operator, $a_{i \sigma}\left(a_{i \sigma}^{\dagger}\right)$ is annihilation (creation) operator for electron with spin $\sigma$ on site $i$, local energy levels $\epsilon_{i}$ are assumed to be independent random variables at different lattice sites. To simplify diagram technique in the following we assume the Gaussian distribution of these energy levels:

$$
\mathcal{P}\left(\epsilon_{i}\right)=\frac{1}{\sqrt{2 \pi} \Delta} \exp \left(-\frac{\epsilon_{i}^{2}}{2 \Delta^{2}}\right)
$$

Parameter $\Delta$ represents here the measure of disorder and this Gaussian random field (with "white noise" correlation on different lattice sites) generates "impurity" scattering and lead to the standard diagram technique for 
calculation of the ensemble averaged Green's functions [21].

Generalized DMFT $+\Sigma$ approach 13 16] extends the standard DMFT [6 8] introducing an additional "external" self-energy $\Sigma_{\mathbf{p}}(\varepsilon)$ (in general case momentum dependent), which is due to some interaction mechanism outside the DMFT. It gives an effective procedure to calculate both single- and two-particle properties [17, 20]. The success of this approach is connected with the choice of the single-particle Green's function in the following form:

$$
G(\varepsilon, \mathbf{p})=\frac{1}{\varepsilon+\mu-\varepsilon(\mathbf{p})-\Sigma(\varepsilon)-\Sigma_{\mathbf{p}}(\varepsilon)},
$$

where $\varepsilon(\mathbf{p})$ is the "bare" electronic dispersion, while the total self-energy neglects the interference between the Hubbard and "external" interaction and is given by the additive sum of the local self-energy $\Sigma(\varepsilon)$ of DMFT and "external" self-energy $\Sigma_{\mathbf{p}}(\varepsilon)$. This conserves the standard structure of DMFT equations [6 8 ]. However, there are two important differences with standard DMFT. At each iteration of DMFT cycle we recalculate the "external" self-energy $\Sigma_{\mathbf{p}}(\varepsilon)$ using some approximate scheme for the description of "external" interaction and the local Green's function is "dressed" by $\Sigma_{\mathbf{p}}(\varepsilon)$ at each step of the standard DMFT procedure.

For "external" self-energy due to disorder scattering entering DMFT $+\Sigma$ cycle below we use the simplest approximation neglecting the diagrams with "intersecting" interaction lines, i.e. the self-consistent Born approximation, For the Gaussian distribution of site energies it is momentum independent and is given by:

$$
\Sigma_{\mathbf{p}}(\varepsilon) \rightarrow \tilde{\Sigma}=\Delta^{2} \sum_{\mathbf{p}} G(\varepsilon, \mathbf{p}),
$$

where $G(\varepsilon, \mathbf{p})$ is the single-particle Green's function (3), while $\Delta$ is the strength of site energy disorder.

To solve the single Anderson impurity problem of DMFT we have employed the reliable algorithm of the numerical renormalization group [22], i.e. the DMFT(NRG) approach..

Within DMFT $+\Sigma$ approach we can also investigate the two-particle properties. In particular, the real part of dynamical (optical)conductivity in $\mathrm{DMFT}+\Sigma$ we have the following general expression [17, 20]:

$$
\begin{array}{r}
\operatorname{Re} \sigma(\omega)=\frac{e^{2} \omega}{2 \pi} \int_{-\infty}^{\infty} d \varepsilon\left[f\left(\varepsilon_{-}\right)-f\left(\varepsilon_{+}\right)\right] \times \\
\times \operatorname{Re}\left\{\phi_{\varepsilon}^{0 R A}(\omega)\left[1-\frac{\Sigma^{R}\left(\varepsilon_{+}\right)-\Sigma^{A}\left(\varepsilon_{-}\right)}{\omega}\right]^{2}-\right. \\
\left.-\phi_{\varepsilon}^{0 R R}(\omega)\left[1-\frac{\Sigma^{R}\left(\varepsilon_{+}\right)-\Sigma^{R}\left(\varepsilon_{-}\right)}{\omega}\right]^{2}\right\},
\end{array}
$$

where $e$ is electronic charge, $f\left(\varepsilon_{ \pm}\right)$- Fermi distribution for $\varepsilon_{ \pm}=\varepsilon \pm \frac{\omega}{2}$, and

$$
\phi_{\varepsilon}^{0 R R(R A)}(\omega)=\lim _{q \rightarrow 0} \frac{\Phi_{\varepsilon}^{0 R R(R A)}(\omega, \mathbf{q})-\Phi_{\varepsilon}^{0 R R(R A)}(\omega, 0)}{q^{2}}
$$

where the two-particle Green's function $\Phi_{\varepsilon}^{0 R R(R A)}(\omega, \mathbf{q})$ contain all vertex corrections from "external" interaction, but do not include vertex corrections from Hubbard interaction. This considerably simplifies calculations of optical conductivity within DMFT $+\Sigma$ approximation, as we have only to solve the single-particle problem determining the local self-energy $\Sigma\left(\varepsilon_{ \pm}\right)$via the DMFT $+\Sigma$ procedure. Non-trivial contribution from non-local correlations enters only via $\Phi_{\varepsilon}^{0 R R(R A)}(\omega, \mathbf{q})$, which can be calculated in appropriate approximation, taking into account only "external" interaction. To obtain the loop contributions $\Phi_{\varepsilon}^{0 R R(R A)}(\omega, \mathbf{q})$, determined by disorder scattering, we can either use the "ladder" approximation for the case of weak disorder, or following Ref. [17], we can use the generalization of the self-consistent theory of localization [23, 24], which allows us to treat the case of strong enough disorder. In this approach conductivity is determined mainly by the generalized diffusion coefficient obtained from the generalization of self-consistency equation [23, 24] of this theory, which is to be solved in combination with DMFT $+\Sigma$ procedure.

In the following we shall consider the three-dimensional system with "bare" semi-elliptic density of states (per elementary cell and one spin projection), which is given by:

$$
N_{0}(\varepsilon)=\frac{2}{\pi D^{2}} \sqrt{D^{2}-\varepsilon^{2}}
$$

with the bandwidth $W=2 D$. All calculations below are done for quarter-filled band $(n=0.5)$. The value of conductivity on all figures will be given in universal units of $\sigma_{0}=\frac{e^{2}}{h a}$ (where $a$ is the lattice spacing).

\section{MAIN RESULTS}

In Fig.1 we show densities of states obtained for $T / 2 D=0.05$ and quarter filling of the band $(n=0.5)$ for different values of attractive $(U<0)$ Fig.1(a) and repulsive $(U>0) \operatorname{Fig} 1$ (b) interaction. It is well known that at half-filling $(n=1)$ density of states of attractive and repulsive Hubbard models just coincide (due to exact mapping of these models onto each other). This is not so when we deviate from half-filling. From Fig[1] we can see that the density of states close to the Fermi level drops with the growth of $U$, both for attraction (Fig 1(a)) and repulsion (Fig $1(\mathrm{~b}))$, but significant growth of $|U|$ in repulsive case leads only to vanishing quasiparticle peak and density of states at the Fermi level becomes practically independent of $U$, while in attractive case the growth of $|U|$ leads to superconducting pseudogap opening at the Fermi level (curve 3 in Fig[1(a)) and for 

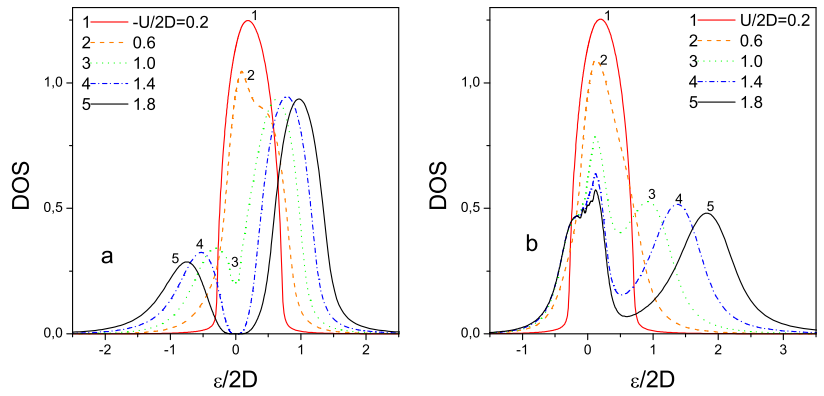

FIG. 1: Densities of states for different values of Hubbard attraction (a) and repulsion (b). Temperature $T / 2 D=0.05$.

$|U| / 2 D>1.2$ we observe the full gap opening at the Fermi level (curves 4, 5 in Fig [1(a)). This gap is not related to the appearance of superconducting state, but is due to the appearance of preformed Cooper pairs, as the temperature for which the results shown in Fig 1 were obtained is larger than superconducting transition temperature (cf. Fig 7 below). Thus we observe the important difference from repulsive case, where the deviation from half-filling leads to metallic state for arbitrary values of $U$, while insulating gap at large $U$ opens not at the Fermi level.

This picture of density of states evolution with the growth of $|U|$ is supported by the behavior of dynamic (optical) conductivity shown in Fig 2. We see that with the growth of $|U|$ Drude peak at zero frequency (curves 1, 2 in Fig 2) is replaced by pseudogap dip (curve 3 in Fig (2) and wide maximum of conductivity at finite frequency, connected with scattering across the pseudogap. The further growth of $|U|$ leads to the appearance of the full gap in optical conductivity due to formation of Cooper pairs (curves 4, 5 in Fig 2).

Similar evolution with growth of $|U|$ is also observed in spectral density. In Fig 3 we show spectral density $A(\varepsilon, \mathbf{p})=-\frac{1}{\pi} \operatorname{Im} G^{R}(\varepsilon, \mathbf{p})$ at the Fermi surface $\left(p=p_{F}\right)$ for different values of attractive interaction $U$. With the growth of $|U|$ a narrow peak in spectral density at the Fermi level (curves 1, 2 in Fig 3) is smeared and with the further growth of $|U|$ the pseudogap dip appears at the Fermi level (curve 3 in Fig 3). At still larger $|U|$ this dip is transformed into the real gap (curves 4, 5 in Fig(3). This behavior of spectral density correlates well with qualitative change (with the growth of $|U|$ ) of distribution function $n\left(\xi_{k}\right)$ (Fig近), defined as:

$$
n\left(\xi_{k}\right)=\int_{-\infty}^{\infty} d \varepsilon A\left(\varepsilon, \xi_{k}\right) f(\varepsilon)
$$

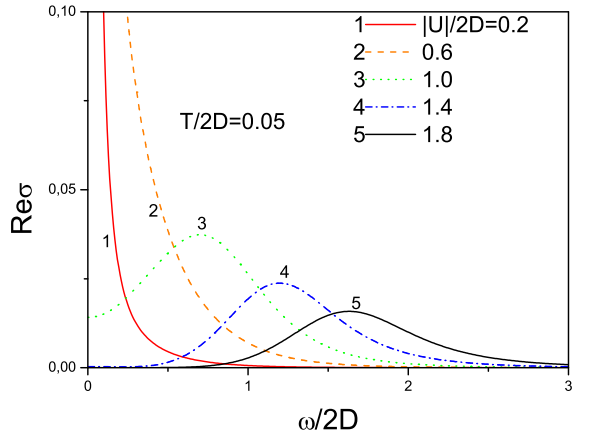

FIG. 2: Optical conductivity for different values of Hubbard attraction. Temperature $T / 2 D=0.05$.

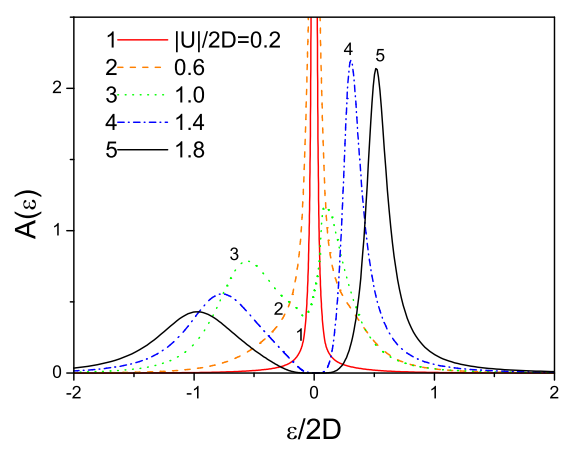

FIG. 3: Spectral density at the Fermi surface for different values of Hubbard attraction. Temperature $T / 2 D=0.05$.

where $\xi_{k}$ represents kinetic energy of electrons. It is seen, that this distribution changes from more or less defined Fermi step-function at small $|U|$ (curves 1, 2 in Fig,4) to effective constant at large values of $|U|$ (curves 4,5 in Fig(4), due to formation of Cooper pairs with binding energy of the order of $|U|$.

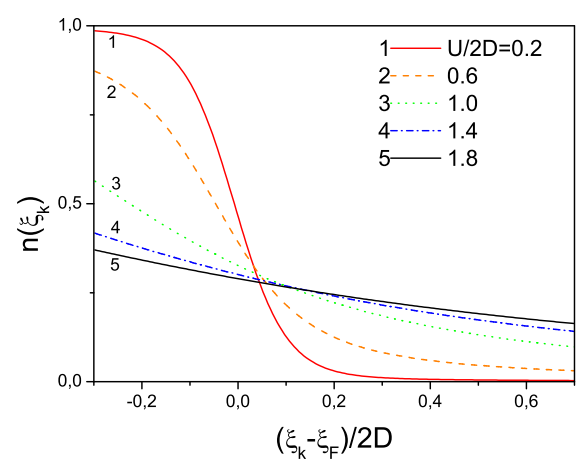

FIG. 4: Distribution function for different values of Hubbard attraction. Temperature $T / 2 D=0.05 . \xi_{F}$ - kinetic energy of electrons at the Fermi surface. 

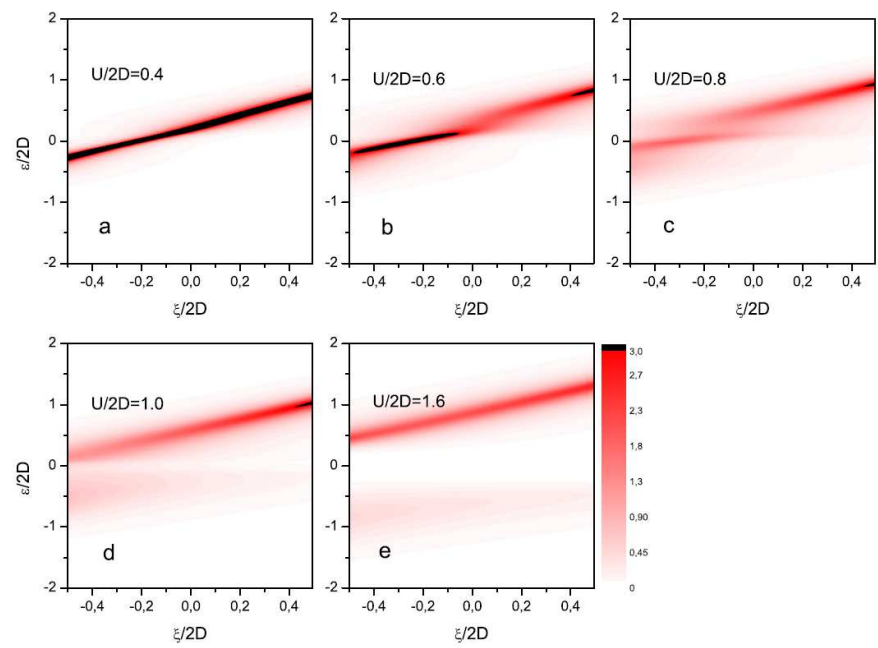

$\xi / 2 D$

$\xi / 2 \mathrm{D}$

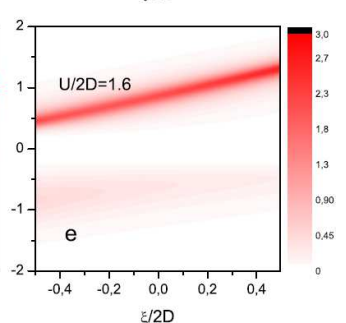

FIG. 5: Spectral density maps for different values of Hubbard attraction. Color represent the intensity of spectral density. Temperature $T / 2 D=0.05$.

The formation of superconducting pseudogap and Cooper pairing gap with the growth of $|U|$ is also well demonstrated by the maps of spectral density, shown in Fig 5 for different values of $U$. Colors represent the intensity of spectral density. We observe that the growth of $|U|$ leads to transformation of initially well defined dispersion of Fig 5(a) to dispersions with pseudogap region, shown in Fig 5 (b,c), which transforms into the real Cooper gap, shown in Fig 5 (d,e) with the further growth of $|U|$.

\section{A. Disorder effects}

In Fig [ 6] she show evolution of the density of states and optical conductivity with changing disorder. At weak enough attraction $(|U| / 2 D=0.8$, Fig $6(\mathrm{a}),(\mathrm{b}))$, we see that the growth of disorder smears density of states, leading to some widening of the band. This smearing masks peculiarities of the density of states due to correlation effects. In particular, quasiparticle peak and "wings" due to upper and lower Hubbard bands observed in the density of states in Fig 6(a) in the absence of disorder completely vanish at strong enough disorder. There are no singularities in the density of states due to Anderson metal-insulator transition, which takes place at $\Delta / 2 D=0.37$ [17], as density of states does not feel Anderson localization. Evolution of optical conductivity with the growth of disorder $\Delta$, shown in Fig $[$ (b), corresponds in general to evolution of density of states. The growth of disorder, while it remains weak enough, (curves 1, 2 in Fig 6(b)), leads to some growth of static conductivity, which is connected with suppression of correlation effects at the Fermi level, noted above (curves 1, 2 in Fig 6(a). The further growth of disorder leads to significant widening of the band and the drop of den- sity of states (curve 3 in Fig 6(a),(b)), which leads to drop of static conductivity. Finally, with the further growth of disorder Anderson localization effects become important. At $T=0$ Anderson transition takes place at $\Delta / 2 D=0.37$ [17]. However, here we consider the case of high enough temperature $T / 2 D=0.05$, so that static conductivity (see curves 4, 5 in Fig 6 (b)) remains finite, though at finite frequencies we clearly observe localization behavior with $\sigma(\omega) \sim \omega^{2}$. At larger value of attractive interaction $|U| / 2 D=1$, the evolution of the density of states and optical conductivity is more or less similar (Fig. 6(c,d) ). However, in the absence of disorder we observe here superconducting pseudogap in the density of states and disorder growth suppresses it, leading both to the growth of the density of states at the Fermi level and appropriate growth of static conductivity. Finally, at still larger attraction $|U| / 2 D=1.6$ (Fig $[$ (e),(f)) in the absence of disorder there is the real Cooper gap in the density of states. This gap is also clearly observed in optical conductivity. With the growth of disorder Cooper gap both in the density of states and conductivity becomes narrower (curves 1-3). Further growth of disorder leads to complete suppression of Cooper gap and restoration of metallic state with finite density of states at the Fermi level and finite static conductivity. This closure of Cooper gap is related to the widening of effective bandwidth $W_{\text {eff }}$ due to disorder, which leads to the diminishing ratio $|U| / W_{\text {eff }}$, which controls the formation of Cooper gap. Situation here is similar to the closure of Mott gap by disorder in repulsive Hubbard model [17]. However, at larger disorder (curve 5 in Fig 6(f)) we clearly observe localization behavior, so that the growth of disorder at $T=0$ will first lead to metallic state (the closure of Cooper gap), while the further growth of disorder will induce Anderson metal-insulator transition. Similar picture is observed for large positive $U$ at half-filling $(n=1)$ [17], where the growth of disorder leads to Mott insulator - correlated metal - Anderson insulator transition.

\section{B. Superconducting transition temperature}

Superconducting transition temperature $T_{c}$ in attractive Hubbard model was studied in a number of papers 9, 10, 12], both from the criterion of instability of normal phase (divergence of Cooper susceptibility) [9] and from the condition of vanishing superconducting order parameter [10, 12]. In Fig. 7 black squares, white circles and white squares show the results of Refs. [9], 10], 12] correspondingly, for the case of quarter-filling $n=0.5$ $(1)$.

\footnotetext{
1 In Ref. 10 it was claimed that $n=0.75$ was considered, but results are obtained practically coincide with those of Ref. [9] obtained for $n=0.5$
} 

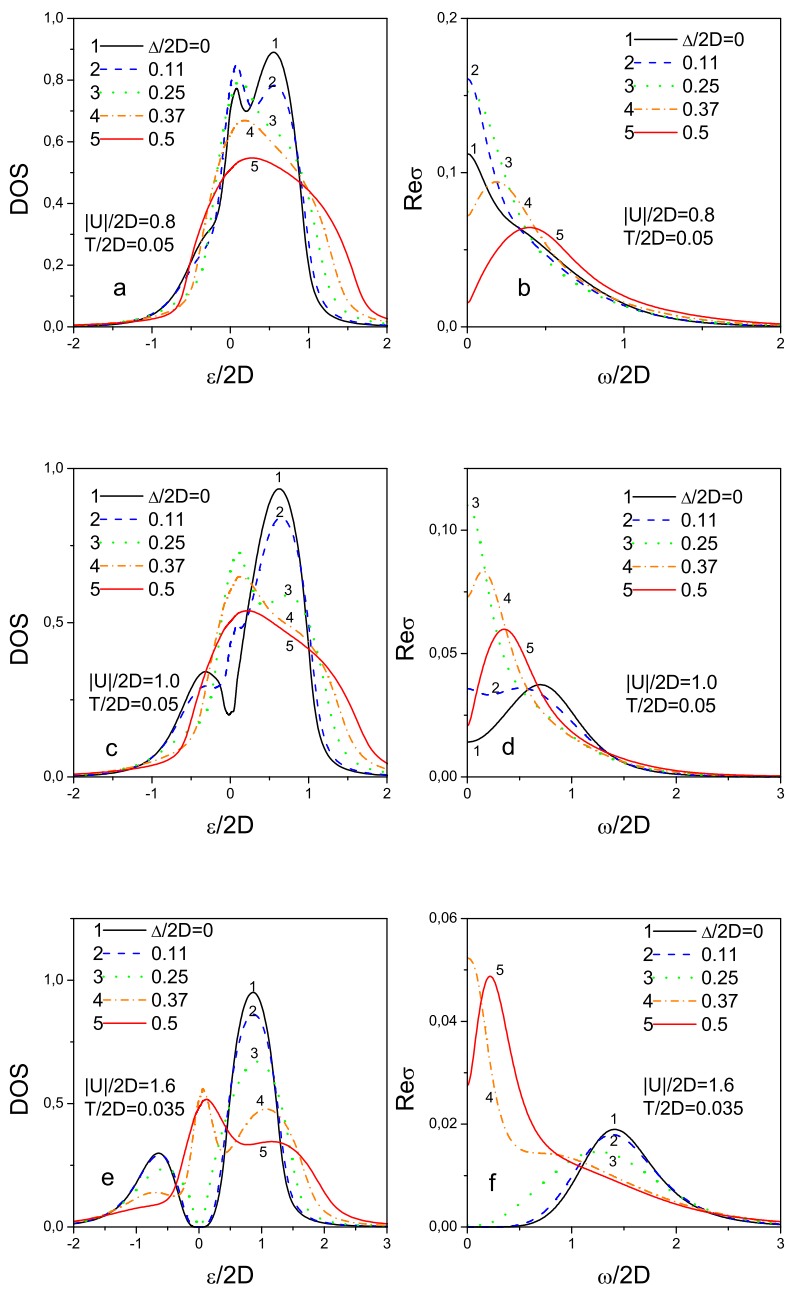

FIG. 6: Evolution of the density of states (left panels) and optical conductivity (right panels) with disorder for different values of $U(|U| / 2 D=0.8-\mathrm{a}, \mathrm{b} ;|U| / 2 D=1$ - c, d; $|U| / 2 D=$ $1.6-\mathrm{e}, \mathrm{f})$.

Actually, the overall picture of $T_{c}$ dependence on $U$ is well approximated by filled circles curve shown in Fig. 7 and obtained from Nozieres - Schmitt-Rink [2] approach, which gives the correct (approximate) description of BCS-BEC crossover. Then for critical temperature $T_{c}$ we have the usual BCS-like equation:

$$
1=\frac{|U|}{2} \int_{-D}^{D} d \varepsilon N_{0}(\varepsilon) \frac{t h \frac{\varepsilon-\mu}{2 T_{c}}}{\varepsilon-\mu},
$$

while the chemical potential for different values of $U$ is to be determined from DMFT calculations (for fixed bandfilling). From Fig. 7 we can see, that in the weak coupling region of $|U| / 2 D \ll 1$ the critical temperature in this approach is close to the usual result of BCS theory (see appropriate curve in Fig (7). For $|U| / 2 D \sim 1$ the critical temperature $T_{c}$ has the maximal value, while for $|U| / 2 D \gg 1$ it drops as $T_{c} \sim 1 /|U|$ [2], because for

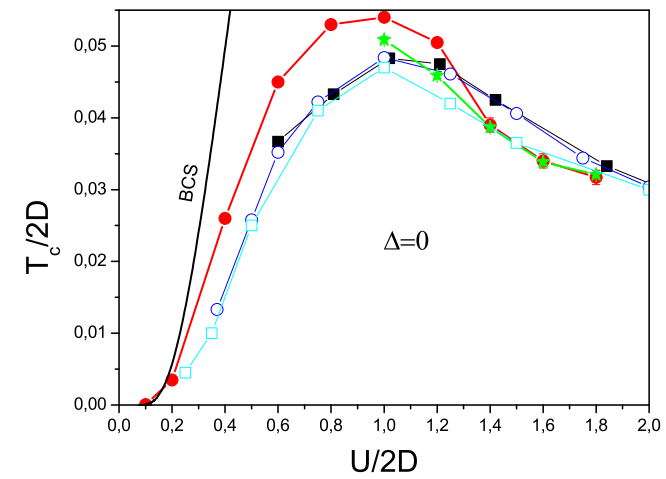

FIG. 7: Dependence of superconducting critical temperature on attractive interaction strength. Black squares, white circles and white squares show the results of Refs. [9], 10], 12] respectively for quarter-filled band with $n=0.5$. Stars represent the results obtained from the criterion of instability of the normal phase. Filled circles show $T_{c}$ obtained Nozieres Schmitt-Rink approximation. Continuous black curve represents the result of BCS theory.

such strong values of attractive interaction the critical temperature is determined by the condition of Bose condensation of preformed Cooper pairs and transfer amplitude of these pairs appears only in the second order of perturbation theory and is proportional to $t^{2} /|U|$ [2]. Stars in Fig:7 show the critical temperature, obtained from the criterion of normal phase instability. For large enough $U$ lowering temperature leads to instability of DMFT(NRG) iteration procedure - at high enough temperatures DMFT(NRG) procedure converges to a single solution, while for temperatures below some critical temperature we observe two different stable solutions for odd or even iterations. We suggest, that this instability of iteration procedure corresponds to the physical instability of the normal phase. Unfortunately, for $|U| / 2 D<1$, the observed instability is rather weak (the difference between the odd and even iterations is too small), thus the accuracy of our calculations is insufficient to determine $T_{c}$ in this way. Surprisingly enough, the results for $T_{c}$ obtained from the approximate approach of Ref. 2] and from instability of DMFT(NRG) cycle are rather close to each other. This is especially surprising for large values of $U / 2 D$ ratio, where pseodigap (or even the real gap) develops in the density of states.

In Fig 8 we show the dependence of critical temperature, obtained from the criterion of normal state instability, on disorder strength $\Delta$ for $|U| / 2 D=1.6$. At small $\Delta$ we observe weak suppression of $T_{c}$ by disorder, which is apparetnly due the general smearing of the density of stated and bandwidth widening by disorder scattering At large enough.disorder we observe the significant growth of $T_{c}$ with the growth of $\Delta$. This is related to the growth of effective bandwith $W_{\text {eff }}$ due to disorder, leading to effective drop of the ration $|U| / W_{\text {eff }}$, controlling the value 


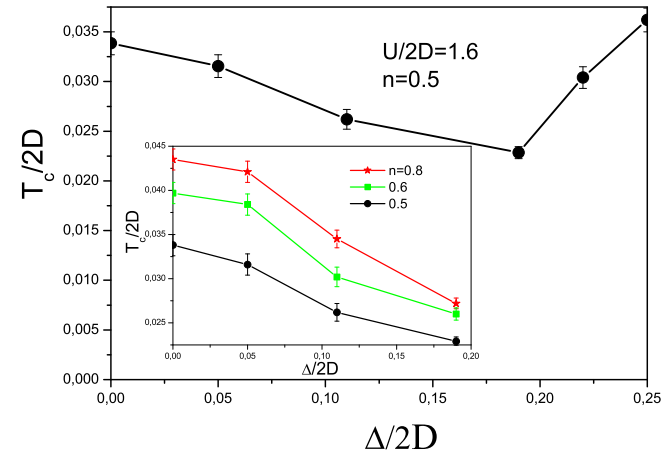

FIG. 8: Dependence of superconducting critical temperature on disorder for $|U| / 2 D=1.6$. At the insert $-T_{c}$ suppression by weak disorder for different values of band-filling: $n=0.5$, $n=0.6, n=0.8$.

of critical temperature in this model. The growth of disorder leads to to the drop of $|U| / W_{\text {eff }}$ from the value of 1.6 at $\Delta=0$ to $|U| / W_{\text {eff }} \sim 1$ for $\Delta / 2 D \sim 0.4$, which leads to the appropriate growth of the critical temperature (cf. Fig 7). This behavior is similar to the growth of the critical value of repulsion in Hubbard model for Mott metal-insulator transition with the growth of disorder (cf. Ref. [17, 18]). The drop of the ratio $|U| / W_{\text {eff }}$ with the growth of disorder does not allow us to guarantee the sufficient accuracy of the values of $T_{c}$ in the case of $|U| / 2 D \sim 1$ for disorder values larger than $\Delta / 2 D=0.11$. For such small values of disorder and for $|U| / 2 D \sim 1$ the critical temperature is weakly suppressed by disorder, similarly to the behavior shown in Fig 8 for the case of $|U| / 2 D=1.6$. At the insert in Fig 8 we show the suppression of the critical temperature by weak disorder for different values of band-filling: $n=0.5, n=0.6, n=0.8$.

\section{CONCLUSIONS}

Within the generalized DMFT $+\Sigma$ generalization of dynamical mean field theory we have studied the properties of the normal (non-superconducting) state of attractive Hubbard model for the wide region of values of onsite attractive interaction $U$. The results for the density of states, spectral density, distribution function and dynamic (optical) conductivity demonstrate the formation of superconducting pseudogap at the Fermi level for intermediate values of coupling strength $|U| / 2 D \sim 1$ and formation of the real Cooper gap in the strong coupling region $|U| / 2 D>1$. The appearance of Cooper gap is related to the formation of compact Cooper pairs at temperatures, which are significantly higher. than the critical temperature of superconducting transition $T_{c}$, which is determined as Bose-condensation temperature of such (preformed) pairs. Within our DMFT $+\Sigma$ approach we have also studied the influence of disorder on the properties of the normal phase. It was shown, that the growth of disorder in the strong coupling region leads to the closure of the Cooper gap and restoration of the metallic state, while in the intermediate coupling region disorder smears superconducting pseudogap and increases the density of states at the Fermi level. In both cases this is related to the general widening of the band (in the absence of $U$ ) by disorder.

We have determined the critical temperature of superconducting transition $T_{c}$ from the condition of instability of the normal phase. Two methods to find such instability were used, demonstrating quantitatively similar results. In the weak coupling region $T_{c}$ is well described by BCS theory, while in the strong coupling region it is related to Bose-condensation of (preformed) Cooper pairs and drops as $1 /|U|$ with the growth of $|U|$, passing through the maximum at $|U| / 2 D \sim 1$. We have also studied the effects of disorder on $T_{c}$. It was shown, that disorder influence of $T_{c}$ is rather weak. In the strong coupling region, e.g for $U / 2 D=1.6$ we observe both weak suppression of critical temperature, as well as some growth of $T_{c}$ with the growth of $\Delta$ for strong enough disorder. In fact, this behavior suggests the validity of Anderson theorem (as was conjectured for BCS-BEC crossover region in Ref. [25]), with changes of $T_{c}$ related to the widening of conduction band by disorder. These results are also consistent with recent lowest order perturbation theory analysis of the effects of disorder throughout BCS-BEC crossover region 26].

This work was partly supported by RFBR grant 1402-00065 and was performed within the Program of Fundamental Research of the Ural Branch of the Russian Academy of Sciences "Quantum macrophysics and nonlinear dynamics" (projects No. 12-П-2-1002, 12-T-21001). 
[1] A. J. Leggett, in Modern Trends in the Theory of Condensed Matter, edited by A. Pekalski and J. Przystawa (Springer, Berlin 1980).

[2] P. Nozieres and S. Schmitt-Rink, J. Low Temp. Phys. 59, 195 (1985)

[3] I. Bloch, J. Dalibard, and W. Zwerger. Rev. Mod. Phys. 80885 (2008)

[4] L.P.Pitaevskii. Usp. Fiz. Nauk 176, 345 (2006) [Physics Uspekhi 49, No. 4, 333 (2006)]

[5] Yen Lee Loh, and Nandini Trivedi. arXiv:1309.4716

[6] Th. Pruschke, M. Jarrell, and J. K. Freericks, Adv. in Phys. 44, 187 (1995).

[7] A. Georges, G. Kotliar, W. Krauth, and M. J. Rozenberg, Rev. Mod. Phys. 68, 13 (1996).

[8] D. Vollhardt in "Lectures on the Physics of Strongly Correlated Systems XIV", eds. A. Avella and F. Mancini, AIP Conference Proceedings vol. 1297 (American Institute of Physics, Melville, New York, 2010), p. 339; ArXiV: 1004.5069

[9] M. Keller, W. Metzner, and U. Schollwock. Phys. Rev. Lett. 86, 46124615 (2001); ArXiv: cond-mat/0101047

[10] A. Toschi, P. Barone, M. Capone, and C. Castellani. New Journal of Physics 7, 7 (2005); ArXiv: cond-mat/0411637v1

[11] J. Bauer, A.C. Hewson, and N. Dupis. Phys. Rev. B 79, 214518 (2009); ArXiv: 0901.1760v2

[12] A. Koga and P. Werner. Phys. Rev. A 84, 023638 (2011); ArXiv: $1106.4559 \mathrm{v} 1$

[13] E.Z.Kuchinskii, I.A.Nekrasov, M.V.Sadovskii. Pisma Zh. eksp. Teor. Fiz. 82, 217 (2005) [JETP Lett. 82, 198 (2005)]; ArXiv: cond-mat/0506215

[14] M.V. Sadovskii, I.A. Nekrasov, E.Z. Kuchinskii, Th. Prushke,V.I. Anisimov. Phys. Rev. B 72, No 15, 155105 (2005); ArXiV: cond-mat/0508585

[15] E.Z. Kuchinskii, I.A. Nekrasov, M.V. Sadovskii. 32, 4/5, 528-537 (2006) [Low Temp. Phys. 32, 398 (2006)]; ArXiv: cond-mat/0510376
[16] E.Z. Kuchinskii, I.A. Nekrasov, M.V. Sadovskii. Usp. Fiz. Nauk 182, 345-378 (2012) [Physics Uspekhi 55, 325 (2012)]; arXiv:1109.2305

[17] E.Z. Kuchinskii, I.A. Nekrasov, M.V. Sadovskii, Zh. Eksp. Teor. Fiz. 133, 670 (2008) [JETP 106, 581 (2008)]; ArXiv: 0706.2618.

[18] E.Z.Kuchinskii, N.A.Kuleeva, I.A.Nekrasov, M.V.Sadovskii. Zh. Eksp. Teor. Fiz. 137, 368 (2010) [JETP 110, 325 (2010)]; ArXiv: 0908.3747

[19] E.Z.Kuchinskii, I.A.Nekrasov, M.V.Sadovskii. Phys. Rev. B 80, 115124 (2009); ArXiv: 0906.3865

[20] E.Z. Kuchinskii, I.A. Nekrasov, M.V. Sadovskii. Phys. Rev. B 75, 115102-115112 (2007); ArXiv: cond-mat/0609404.

[21] A.A. Abrikosov, L.P. Gorkov, I.E. Dzyaloshinskii. Quantum Field Theoretical Methods in Statistical Physics. Pergamon Press, Oxford 1965; M. V. Sadovskii. Diagrammatics. World Scientific, Singapore 2006.

[22] R. Bulla, T.A. Costi, T. Pruschke, Rev. Mod. Phys. 60, 395 (2008).

[23] D. Vollhardt and P. Wölfle. Phys. Rev. B 22, 4666-4679 (1980); Phys. Rev. Lett. 48, 699 (1982); in Anderson Localization, eds. Y. Nagaoka and H. Fukuyama, Springer Series in Solis State Sciences, vol. 39, p.26. Springer Verlag, Berlin 1982.

[24] M.V. Sadovskii. The Theory of Electron Localization in Disordered Systems. Soviet Scientific Reviews - Physics Reviews, ed. I.M. Khalatnikov, vol. 7, p.1. Harwood Academic Publ., NY 1986; A.V. Myasnikov., M.V. Sadovskii. Fiz. Tverd. Tela 24, 3569 (1982) [Sov. Phys.-Solid State 24, 2033 (1982) ]; E.A. Kotov, M.V. Sadovskii. Zs. Phys. B 51, No 1, 17 (1983)

[25] A.I. Posazhennikova and M.V. Sadovskii. Pisma Zh. Eksp. Teor. Fiz. 65, 258 (1997) [JETP Letters 65, 270 (1997)]

[26] F. Palestini, G.C. Strinati. arXiv:1311.2761 